\title{
Circuit
}

Musiques contemporaines

\section{Index des auteurs}

Volume 1, numéro 1 (1990) à volume 19, numéro 3 (2009)

Volume 20, numéro 1-2, 2010

La musique contemporaine d'hier à demain

URI : https://id.erudit.org/iderudit/039642ar

DOI : https://doi.org/10.7202/039642ar

Aller au sommaire du numéro

Éditeur(s)

Les Presses de l'Université de Montréal

ISSN

1183-1693 (imprimé)

1488-9692 (numérique)

Découvrir la revue

Citer ce document

(2010). Index des auteurs : volume 1, numéro 1 (1990) à volume 19, numéro 3 (2009). Circuit, 20(1-2), 39-52. https://doi.org/10.7202/039642ar d'utilisation que vous pouvez consulter en ligne.

https://apropos.erudit.org/fr/usagers/politique-dutilisation/ 


\section{Index des auteurs}

Volume 1, numéro 1 (1990) à volume 19, numéro 3 (2009)

\author{
AlePIN, Suzanne \\ "Répertoire des analyses d'œurres de \\ compositeurs québécois", vol. 11, n 1 \\ (2000), p. 87-104.

\section{Ambrosini, Claudio} \\ "À propos de Fausto Romitelli: \\ Le Corps électrique", vol. 17, $\mathrm{n}^{\circ} 1$ (2007), \\ p. 121-124.
}

\section{ARCURI, Serge}

"Ondes de choc", vol. 4, n 1-2 (1993), p. $165-166$.

\section{BAIL, Louise}

"Introduction à Kopernikus: pistes de réflexion autour du sacré", vol. 18, n 3 (2008), p. 9-26.

\section{Baillargeon, Stéphane}

"Pour résoudre une dissonance", vol. 3, nº 1 (1992), p. 93-96.

\section{BAKER, Robert A.}

"Spaces and Places of Opera", vol. 17, $n^{\circ} 3$ (2007), p. 57-63.

\section{BARDEZ, Jean-Michel}

"La classe de Messiaen de Jean Boivin", vol. 9, nº 1 (1998), p. 27-40.

\section{BARRIÈRE, Françoise}

"De Bourges: un paysage complexe et nuancé", vol. 4, no 1-2 (1993), p. 37-46.

"Où en est la musique électroacoustique aujourd'hui?", vol. 13, n⿳⺈ 3 (2003), p. 9-17.

\section{BARRIÈRE, Jean-Baptiste}

"À propos de l'IRCAM : avatars d'une critique culturelle", vol. 10, n 2 (1999), p. 59-72.

"De Paris: notes critiques", vol. 4, no 1-2 (1993), p. 47-50.

\section{Beaucage, Réjean}

"Mutek — flou artistique?", vol. 13, $\mathrm{n}^{\circ} 1$ (2002), p. 35-40.

"Avant-propos. Les métissages alchimiques de Frank Zappa (19401993)", vol. 14, n 3 (2004), p. 5-7.

"Différents sons de cloches. La parole aux artisans", vol. 11, $n^{\circ} 3$ (2001), p. 11-19.

"Elektra 2002. Le poids du passé", vol. 13, $\mathrm{n}^{\circ} 3$ (2003), p. 75-83.

"Empreintes Digitales, une étiquette qui laisse des traces", vol. 12, n० 3 (2002), p. 63-69.

"Établir les contacts", vol. 13, n 3 (2003), p. 85-96.

"Festival Elektra 2001 ", vol. 12, n 2 (2002), p. 81-98.

«Frank Zappa dans l'univers des musiques sérieuses: un parfait étranger?", vol. 14, n 3 (2004), p. 9-31. "Interprète et créateur. Entretien avec Benjamin Carat", vol. 15, n 1 (2004), p. 65-72.

"Le cas d'Espaces sonores illimités", vol. 17, no 3 (2007), p. 39-44.
"Le courant électrique dans Montréal/ Nouvelles Musiques", vol. 14, no 2 (2004), p. 29-34.

"Portrait de Réseaux des arts médiatiques", vol. 16, n 3 (2006), p. 107-115.

"Quelques éléments biographiques des fondateurs de Réseaux", vol. 16, $n^{\circ} 3$ (2006), p. 117-118.

"Réinventer le rituel du concert. Entretien avec Marc Couroux", vol. 15, $\mathrm{n}^{\circ} 1$ (2004), p. 57-63.

"Voir la musique aujourd'hui?", vol. 16, nº 3 (2006), p. 43-50.

"La $25^{e}$ édition du FIMAV. Actualité de la musique... actuelle", vol. 18, no 2 (2008), p. 89-92.

\section{Beaucage, Réjean et Panneton,} Isabelle

Présentation du numéro «Interpréter la musique (d')aujourd'hui", in vol. 15, $n^{0} 1$ (2004), p. 7-8.

\section{Bergeron, Carol}

"Quelques disques de musique actuelle", vol. 6, nº 2 (1995), p. 69-71.

\section{Berio, Luciano, Boulez,}

Pierre et Pousseur, Henri

(Jean-Jacques Nattiez et Marc Chenard [éd.])

"Table ronde de 1963, journées des 16, 18, 23 et 25 juillet", vol. 15, n ${ }^{\circ} 3$ (2005), p. $23-52$. 


\section{BERTONCINI, Mario}

"Dialogue n 1 . Presque une préface", Véronique Robert (trad.), vol. 15, $\mathrm{n}^{0} 1$ (2004), p. 101-104.

\section{Bıró, Dániel Péter}

"Remembering and Forgetting: Lizkor VeLishkoach for String Quartet, after Schubert", vol. 18, nº 2 (2008), p. 39-60.

\section{BissonnetTe, Lise}

"Nos dissonances", vol. 7, nº 1 (1996), p. 79-82.

«Ruptures", vol. 7, nº 1 (1996), p. 13-15.

\section{BissonNETTE, Lise et al.}

"Une table ronde au FM de RadioCanada - Qui a peur de la musique contemporaine?", vol. 7, nº 1 (1996), p. $40-51$

\section{BlackBurn, Philip}

"American Composers Forum. Se réseauter ou au-delà de la pige d'un soir dans le ghetto de la nouvelle musique", vol. 14, nº 2 (2004), p. 67-76

\section{BlumRöDER, Christoph von}

"S'orienter selon Hermann Hesse", Martin Kaltenecker et Elsa Rieu (trad.), vol. 19, $n^{\circ} 2$ (2009), p. 11-34.

\section{BoIvin, Jean}

"Les élèves inscrits à la classe de composition de Garant", vol. 7, n ${ }^{\circ} 2$ (1996), p. 53-55.

"La classe de composition de Serge Garant, ou le sentier de la lucidité", vol. 7, no 2 (1996), p. 37-52.

"Le festival Présences 99. Une fenêtre ouverte sur la nouvelle réalité musicale québécoise", vol. 10, nº 2 (1999), p. 39-50.

"Le quatuor, le compositeur et le musicologue - Fable", vol. 11, $\mathrm{n}^{\circ} 2$ (2000), p. 69-72.
"Éditorial" [du numéro sur Serge

"Le Traité de rythme, de couleur, et d'ornithologie d'Olivier Messiaen (tomes I, II, III et IV)", vol. 9, no 1 (1998), p. 17-25.

"Les musiques classique, moderne et contemporaine larguées par la radio publique - Le cas d'Espace musique", vol. 16, $n^{\circ} 3$ (2006), p. 95-105.

Éditorial [du numéro "Les Racines de l'identité»], vol. 10, nº 2 (1999), p. 5-8.

\section{BONNET, Antoine}

"De l'idée à l'œuvre. Figures, fonctions, formes, langage dans la Notation I pour orchestre de Pierre Boulez", vol. 17, nº 1 (2007), p. 49-64.

\section{Borıo, Gianmario}

"L'analyse musicale comme processus d'appropriation historique - Webern à Darmstadt", Anne Giannini (trad.), vol. $15, n^{\circ} 3$ (2005), p. 87-122.

\section{BOUdREAU, Walter}

"Circuits-multiples - Circa 1970-1980: les années hard core (faire "respirer" la musique)", vol. 8, n 1 (1997), p. 31-42.

"Panser la musique aujourd'hui", vol. 3, nº 1 (1992), p. 23-27.

\section{Bouhalassa, Ned}

"Électroniquoi? Chronique de la naissance d'une nouvelle constellation sonore", vol. 13, nº 1 (2002), p. 27-34.

\section{BoulEZ, Pierre}

"Zukunftsmusik? Avenir de la musique1?", vol. 3, n 1 (1992), p. 7-21.

\section{Boulez, Pierre, et NatTiez, Jean-Jacques}

"In memoriam: Christian Bourgois (1933-2007)", vol. 19, nº 2 (2009), p. 99-101.

\section{Bouliane, Denys}

"À propos... québécitude, musique et postmodernité", vol. 3, nº 2 (1992), p. 69-79.
"L'image fécondante du silène. D'une incursion du doute, du rire et de l'ironie dans le musical: souvenirs, réflexions, "autoréflexions" ", vol. 9, $\mathrm{n}^{\circ} 2$ (1998), p. 25-60.

\section{BOURION, Sylveline, et FORGET, Georges}

"Identité, création et mort contemporaines", vol. 15, n 2 (2005), p. $37-53$.

\section{BRADY, Tim}

"Les confessions d'un autodidacte un peu trop scolarisé", vol. 10, nº 1 (1999), p. $53-62$.

\section{BreAult, Marie-Hélène \\ "Kathinkas Gesang als Luzifers}

Requiem - Réflexions d'une interprète sur une œuvre mixte", vol. 19, $\mathrm{n}^{\circ} 2$ (2009), p. 87-97.

\section{Buch, Esteban}

"L'avant-garde musicale à Buenos Aires: Paz contre Ginastera ", vol. 17, $\mathrm{n}^{0} 2$ (2007), p. 11-33.

\section{CAGE, John}

"Canada, November 1989, Montreal Mesostic de John Cage à Guy Soucie", vol. 8, no 2 (1997), p. 18-19.

\section{Calon, Christian}

"Aujourd'hui = hier + demain", vol. 4, no 1-2 (1993), p. 127-137.

\section{CAmpos, Rémy}

"Traces d'écoutes - Sur quelques tentatives historiennes de saisie du corps de la musique", vol. 14, nº 1 (2004), p. 7-17.

\section{CARON, Sylvain}

"Raynald Arseneault: musique pour l'âme", vol. 8, n 1 (1997), p. 49-53.

\section{Cera, Andrea}

"Noir Miroir : ambiguïtés topographiques, sociales et interactives de la musique", vol. 17, $n^{0} 3$ (2007), p. 29-38. 


\section{Champagne, Éric}

"L'opéra pour enfants ou la quête d'identité. Commentaire critique sur L'arche, d'Isabelle Panneton et Anne Hébert", vol. 16, nº 2 (2006), p. 83-91. "La musique dans l'espace urbain: le cas des Symphonies portuaires de Montréal", vol. 17, n 3 (2007), p. 65-74. "Une saison effervescente pour l'opéra contemporain à Montréal", vol. 16, $n^{\circ} 2$ (2006), p. 93-102.

\section{ChANG, Leiling}

"L'odyssée musicale afrocubaine

- Entre art et politique, entre nationalisme et panaméricanisme", vol. 17, nº 2 (2007), p. 93-106.

\section{Charles, Daniel}

"Composer l'imprévisible (Notes sur John Cage et les années cinquante)", vol. $8, n^{\circ} 2$ (1997), p. 71-80

Charuest, Yves, Béland, Pierre, ThibodeAu, Martin, Dugré, François, et RATTÉ, Michel "Vente de feu de la musique contemporaine chez les intellectuels médiatiques", vol. 7, nº 1 (1996), p. $63-65$.

\section{CochINI, Roger,}

Musique électroacoustique et service public", vol. 13, n 3 (2003), p. 51-58.

\section{COPELAND, Darren}

"Survival Strategies for Electroacoustic Music", vol. 13, n 3 (2003), p. 59-65.

\section{CôTÉ, Michel F}

"Considérations en provenance de Sirius", vol. 19, no 2 (2009), p. 57-62.

«Ruer dur et faire entendre (Dix fragments sur l'art politique de Frank Zappa)", vol. 14, n³ 3 (2004), p. 91-101. "Cirque nominatif", vol. 6, n 2 (1995), p. $27-29$.

\section{Couroux, Marc}

"Dompter la mer sauvage. Réflexions sur Evryali de Iannis Xenakis", vol. 5, $n^{\circ} 2$ (1994), p. 55-67.

"Regard sur quatre créateurs", vol. 10, n 1 (1999), p. 17-40.

"Some Ideas about Viewer Re-Mobilization from a Practice-inProgress", vol. 16, no 3 (2006), p. 83-93.

\section{Couture, Ariane}

"Portrait de Michel Longtin.

Biographie et liste d'œuvres", vol. 14, $n^{\circ} 2$ (2004), p. 83-91.

\section{Culver, Andrew}

"En pensant à Cage avec le Québec en tête", vol. 8, nº 2 (1997), p. 45-51.

"Mesotic for Mario", vol. 15, nº 1 (2004), p. 96-100.

"MNM : A Collective Love Letter by and to Musicians", vol. 14, n 2 (2004), p. 23-28.

\section{Current, Brian}

"A Young Person's Guide for audiences of all ages", vol. 16, n 2 (2006), p. $69-72$.

"The Lateral Extension of the Orchestra”, vol. 13, nº 3 (2003), p. 117123.

\section{DAL FARRA, Ricardo}

"The Southern Tip of the

Electroacoustic Tradition", vol. 17, $\mathrm{n}^{0} 2$ (2007), p. 65-72

\section{DANIELSON, Janet}

"Canadian Women Composers in Modernist Terrain - Violet Archer, Jean Coulthard and Barbara Pentland", vol. 19, nº 1 (2009), p. 57-70.

\section{DaOUST, Yves}

"Entretien de Yves D. avec lui-même", vol. 8, nº 1 (1997), p. 19-25.

"Entrevue avec Jean-François Denis", vol. 13, no 1 (2002), p. 57-62.
«Pensée concrète, démarche abstraite", vol. 11, n 1 (2000), p. 35-42.

\section{DARBON, Nicolas}

"Le concept de complexité dans Cinquante ans de modernité musicale de Célestin Deliège", vol. 16, n 1 (2006), p. 45-57.

\section{DAvoIne, Françoise}

"L'aventure du disque de musique québécoise - Bilan d'une décennie et perspective d'avenir", vol. $1, n^{\circ} 2$ (1991), p. 81-91.

"Sérieuse, audacieuse, bienheureuse... Généreuses! Rencontre avec Isabelle Panneton, Marie Pelletier et Ana Sokolovic", vol. 10, nº 1 (1999), p. 73-81.

\section{De MÉdicIs, François}

"Boulez analyste", vol. 3, n`1 (1992), p. 77-81.

\section{De Mestral, Charles}

"Souvenirs vivants de Mario Bertoncini ", vol. 15, n 1 (2004), p. $93-95$

\section{Delalande, François}

"Towards an Analysis of Compositional Strategies", Timothy Barnard (trad.), vol. 17, $\mathrm{n}^{\circ} 1$ (2007), p. 11-26.

\section{Delbecque, Yannick}

"Portrait de Michel Longtin. Entretien", vol. 14, no 2 (2004), p. 113-121.

\section{DelièGE, Célestin}

"Dans les dédales du "tour de force" ", vol. 1, n ${ }^{0} 1$ (1990), p. 63-73

«Dialectiques et ruses de l'histoire (Comme un bref retour sur soimême...)", vol. 16, nº 1 (2006), p. $89-95$.

\section{DENIS, Jean-Pierre}

"Perceptions", vol. 11, n 3 (2001), p. $39-52$. 


\section{DENUT, Éric}

"Orphée ressuscité? Un panorama de l'opéra contemporain en Europe" vol. $12, n^{\circ} 2$ (2002), p. 9-19.

\section{Derome, Jean}

"Qu'est-ce que ça veut dire, musique actuelle?", vol. 6, no 2 (1995), p. 15-17

\section{DESJARDins, Jacques}

"Brian Cherney et Bruce Mather. La clairvoyance du compositeur solitaire", vol. 10, nº 2 (1999), p. 17-28.

\section{DesjaRdins, Pierre}

"Échec total de la musique contemporaine", vol. 7, n 1 (1996),

p. 51-54.

"La musique contemporaine s'est embrouillée", vol. 7, nº 1 (1996), p. $70-73$

\section{Dhomont, Francis}

"L'électroacoustique sur orbite", vol. 3, no 1 (1992), p. 109-118.

"Le postmodernisme en musique Aventure néo-baroque ou nouvelle aventure de la modernité?", vol. 1, $\mathrm{n}^{0} 1$ (1990), p. 27-47.

"Petite apologie de l'art des sons fixés", vol. 4, n 1-2 (1993), p. 55-66.

"Repensons la musique aujourd'hui", vol. 3, n 1 (1992), p. 27-32.

Éditorial [du numéro sur l'électroacoustique au Québec], vol. 4, $n^{0}$ 1-2 (1993), p. 5-7.

\section{Donin, Nicolas}

" "Assimiler la musique contemporaine m'a permis de mesurer le décalage entre elle et moi."- Entretien avec Chen Qigang", vol. 12, nº 3 (2002), p. 9-22.

" "Une calligraphie est pour moi comme une partition inédite." Entretien avec Wen De-Qing", vol. 12, $n^{0} 3$ (2002), p. 23-33.
"Compositeurs chinois en Europe. Entretiens avec Chen Qigang et Wen De-Qing", vol. 12, n 3 (2002), p. 9-33.

"Le concert, objet musicologique? Compte rendu du livre Le concert. Enjeux, fonctions, modalités (L'Harmattan)", vol. 12, no 1 (2001), p. 87-95.

"Le premier grand bilan analytique de l'itinéraire de Gérard Grisey: Gérard Grisey, Fondements d'une écriture, par Jérôme Baillet", vol. 11, nº 3 (2001), p. 55-58.

"Le travail de la répétition - Deux dispositifs d'écoute et deux époques de la reproductibilité musicale, du premier au second après-guerre", vol. 14, nº 1 (2004), p. 53-85.

"Les cours d'été de Darmstadt, du mythe au chantier", vol. 15, n 3 (2005), p. 5-17.

"Livrer un opéra, opérer un livre : À propos de la création de Manuscrit trouvé à Saragosse de José Evangelista et Alexis Nouss", vol. 12, n 2 (2002), p. 73-79.

"Post-scriptum bibliographique", vol. 14, nº 1 (2004), p. 103-106.

"Pour une "écoute informée" de la musique contemporaine - Quelques travaux récents", vol. 16, nº 3 (2006), p. 51-64.

"Qui écoute?", vol. 13, nº 2 (2003), p. 5-10.

Éditorial [du numéro "Qui écoute? $2 "]$, vol. 14, no 1 (2004), p. 5-6.

\section{Donin, Nicolas, et Goldman, Jonathan}

"Souvenirs de Darmstadt, mode d'emploi ", vol. 15, n 3 (2005), p. 19-22.

\section{Donin, Nicolas, et Theureau, Jacques}

"Ateliers en mouvement. Interroger la composition musicale aujourd'hui", vol. 18, no 1 (2008), p. 5-14.
"L'atelier d'un réalisateur en informatique musicale. Entretien avec Gilbert Nouno", vol. 18, nº 1 (2008), p. 31-38.

"La coproduction des œuvres et de l'atelier par le compositeur - À partir d'une étude de l'activité créatrice de Philippe Leroux entre 2001 et 2006 ", vol. $18, n^{\circ} 1$ (2008), p. 59-71.

\section{DUCHESNEAU, Michel}

Avant-propos [du numéro "La route de soi "], vol. 12, $n^{\circ} 3$ (2002), p. 5-6.

Avant-propos [du numéro "Carte d'identités"], vol. 15, nº 2 (2005), p. 5.

Avant-propos [du numéro "Écrire I'histoire au $20^{\text {e }}$ siècle"], vol. $16, \mathrm{n}^{\circ} 1$ (2006), p. 5-7.

Avant-propos [du numéro "Henri Pousseur - Visages"], vol. 12, $\mathrm{n}^{\circ} 1$ (2001) , p. 5-6.

Avant-propos [du numéro "Opéra aujourd'hui»], vol. 12, n 2 (2002), p. 5-7.

Avant-propos [du numéro "Perceptions"], vol. 11, n 3 (2001), p. 5-9.

Avant-propos [du numéro "Interpréter la musique (d')aujourd'hui"], vol. 15, $n^{0} 1$ (2004), p. 5-6.

Éditorial [du numéro "Le Quatuor selon Schafer "], vol. 11, $\mathrm{n}^{\circ} 2$ (2000), p. 5-6.

Éditorial [du numéro "Montréal/ Nouvelles musiques"], vol. 14, $\mathrm{n}^{\circ} 2$ (2004), p. 5-7.

"Le miroir aux alouettes" [Éditorial du numéro "Musique de création et jeunes publics"], vol. 16, $\mathrm{n}^{\circ} 2$ (2006), p. 5-19.

"Montréal/Nouvelles Musiques: perspectives", vol. 14, n' 2 (2004), p. 9-21. 


\section{DuFOuRT, Hugues}

"La philosophie de la musique de Deliège", vol. 16, nº 1 (2006), p. 59-64.

Dun, Tan

"Comments by Tan Dun on his Music", Shawn Mativetsky (éd.), vol. 12, $n^{\circ} 3$ (2002), p. 35-43.

\section{DunsBY, Jonathan}

"What kind of history is The Cambridge History of Twentieth-Century Music?", vol. 16, no 1 (2006), p. 135-142.

\section{DUPONT, Maÿlis}

"Compte rendu: Penser l'ouvre musicale au xxe siècle: avec, sans ou contre l'Histoire? (Kaltenecker et Nicolas [éd.]", vol. 17, nº 2 (2007), p. 121-127.

\section{Époque, Martine}

"André Prévost et la danse: une rencontre fructueuse", vol. 12, $\mathrm{n}^{0} 1$ (2001), p. 101-102.

\section{Evangelista, José}

"Clés du Manuscrit trouvé à Saragosse", vol. 12, nº 2 (2002), p. 53-68.

"Mon expérience privée du quatuor à cordes", vol. 11, n² 2 (2000), p. 67-68.

"Pourquoi composer de la musique monodique", vol. 1, nº 2 (1991),

p. 55-70.

"Un vieux moderne", vol. 3, no 1 (1992), p. 32-34.

\section{EVERETT-GREEN, Robert}

"L'œuvre maîtresse de Taruskin", Hélène Panneton (trad.), vol. 16, nº 1 (2006), p. 129-133.

\section{FeneYrou, Laurent}

"...écoute cet instant... Helmut Lachenmann / Luigi Nono", vol. 13, $\mathrm{n}^{\circ} 2$ (2003), p. 51-79.

\section{Ferguson, Sean}

"De-Composing Tristan Murail: The Collected Writings, 1980-2000", vol. 17, n० 1 (2007), p. 115-120.

"Review of R Murray Schafer: 'String Quartets Nos. 1-7' and André Prévost: 'String Quartets Nos. 2-4", vol. 11, $n^{\circ} 2$ (2000), p. 90-93.

\section{FilteAu, Pierre}

"Un historique des formats de reproduction", vol. 16, $\mathrm{n}^{\circ} 3$ (2006), p. 17-31.

\section{FISETTE, Jean}

"Claude Gauvreau: musicien, dramaturge, poète", vol. 3, no 2 (1992), p. 7-19.

\section{Fox, Christopher}

[Souvenirs de Darmstadt], vol. 15, $\mathrm{n}^{\circ} 3$ (2005), p.67-69.

\section{GALAISE, Sophie}

"Note éditoriale", vol. 10, nº 1 (1999), p. 7-10.

GALAISE, Sophie (dossier réuni par) " "Désormais, nous écartèlerons son visage..." Neuf compositeurs québécois réagissent aux propos de Pierre Boulez", vol. 3, nº 1 (1992), p. 23-43.

\section{GALAISE, Sophie et al.}

"Xenakis au Québec. Chronologie et repères", vol. 5, n 2 (1994), p. 77-81.

\section{GALAISE, Sophie et BoIvin, Jean} "Les concerts de musique "sérieuse" et quelques autres", vol. 1, n 2 (1991), p. 31-34.

GALAISE, Sophie et RIVESt, Johanne "Compositeurs québécois - Chronique d'une décennie (1980-1990)", vol. 1, no 1 (1990), p. 83-97.
GALATY, James

"Layers of Illusions: John Rea's Hommage à Vasarely", vol. 17, no 2 (2007), p. 107-120.

\section{GALLET, Bastien}

"Composer des étendues, projeter des images. Deux pratiques de l'art sonore", vol. 17, n' 3 (2007), p. 21-27.

\section{GANDINI, Gerardo}

"Entretien avec Gerardo Gandini. "Dans l'Argentine d'aujourd'hui, la composition est devenue une chose solitaire" ". Entretien avec Antonieta Sottile, vol. 17, $n^{\circ} 2$ (2007), p. 73-81.

\section{GANN, Kyle}

"Que signifie et ne signifie pas la musique des femmes?", Guy Marchand (trad.), vol. 19, $n^{\circ} 1$ (2009), p. 9-13.

\section{GATTI, Nathalie}

"Frank Zappa - l'esthétique d'un nomade", vol. 14, nº 3 (2004), p. 45-63.

\section{GAUTHIER, Mario}

"Du sillon fermé au sillon ouvert. Quelques préalables et une discographie", vol. 4, nº 1-2 (1993), p. $145-156$

\section{Gervais, Raymond}

"Où êtes-vous donc, John Cage?", vol. 8, n 2 (1997), p. 33-38

"Quelle(s) musique(s)?", vol. 6, no 2 (1995), p. 7-9.

\section{GIBSON, Benoît}

"La théorie et l'œuvre chez Xenakis. Éléments pour une réflexion", vol. 5, $n^{\circ} 2$ (1994), p. 41-54.

\section{GILBERT, Nicolas et MCKINLEY, Maxime \\ "Montréal/Nouvelles Musiques: compte rendu ", vol. 14, $n^{\circ} 2$ (2004), p. 35-47.}




\section{GILBERT, Nicolas}

"Chute/Parachute de Michel

Gonneville - Pensée sérielle, écriture postmoderne (ou l'inverse)", vol. 19, $n^{0} 3$ (2009), p. 91-108.

"Le Forum 2002 du NEM ", vol. 13, n 3 (2003), p. 97-102.

"Une visite à l'atelier de José Evangelista", vol. 18, n 1 (2008), p. 21-26.

\section{GILMORE, Bob}

"Claude Vivier and Karlheinz Stockhausen - Moments from a Double Portrait”, vol. 19, nº 2 (2009), p. $35-49$.

\section{GINGRAS, Claude et les Mélodistes indépendants}

"À bas la musique "contemporaine"! Quatre compositeurs s'unissent pour promouvoir une musique où la mélodie domine", vol. 7, nº 1 (1996), p. $55-60$.

\section{GIROUARD, Olivier}

"Portrait de Michel Longtin. À propos de Quaternions", vol. 14, n 2 (2004), p. 93-112.

\section{GIROUDON, James}

"Un état des lieux, vivifiant", vol. 1, $n^{\circ} 2$ (1991), p. 9-17.

\section{GoLDMAN, Jonathan}

"Introduction - Quelques sillons...", vol. $16 n^{\circ} 3$ (2006), p. 9-15.

" Musimarch 2002": Causeries and Round Table Discussion. Chronicle of a Partially Imagined Event”, vol. 12, $\mathrm{n}^{\circ} 3$ (2002), p. 45-55.

"“ Symphonie du millénaire". The Sacred in the Profane", vol. 11, $\mathrm{n}^{\circ} 3$ (2001), p. 33-38.

"Autour du concept de "recherche musicale" ", vol. 10, no 2 (1999), p. $55-58$
"Bibliographie sélective des articles et des ouvrages musicologiques sur R. Murray Schafer", vol. 11, n 2 (2000), p. $77-82$.

"De l'autre Amérique à la nôtre. Un regard déboussolant", vol. 17, n 2 (2007), p. 5-9.

"Défense d'Adorno contre ses enthousiastes - Compte rendu de Sound Figures par Theodor W. Adorno", vol. 11, nº 2 (2000), p. 85-90.

"Éditorial. De la contemporanéité de l'arrangement", vol. 18, nº 2 (2008), p. 5-9.

"Éditorial. De la musique, de la contemporanéité et du plaisir", vol. 16, $\mathrm{n}^{0} 3$ (2006), p. 5-8.

"Éditorial. L'idée avant l'œuvre", vol. 17, no 1 (2007), p. 5-10.

“Interview with Joseph Petric", vol. 15, $n^{0} 1$ (2004), p. 73-85.

"Introduction. Compositrices sur la scène contemporaine", vol. 19, $\mathrm{n}^{0} 1$ (2009), p. 5-8.

"Pierre Boulez, théoricien de l'écoute", vol. 13, nº 2 (2003), p. 81-91.

"Premiers Kontakte", vol. 19, n ${ }^{\circ} 2$ (2009), p. 5-9.

"Voyage au bout de l'œuvre Prologue au numéro", vol. $18, \mathrm{n}^{\circ} 3$ (2008), p. 5-7.

\section{GonNEVILLE, Michel}

"La seconde apothéose de Rameau: au-delà de la polémique", vol. 12, nº 1 (2001), p. 45-70.

"Ce qui doit sortir", vol. 3, nº 1 (1992), p. 35-40.

"D'ici et d'ailleurs", vol. 6, nº 2 (1995), p. 71-80.

"Faire face à la musique", vol. 7, n ${ }^{\circ} 1$ (1996), p. 19-24.

"Henri Pousseur: Couleurs croisées et La seconde apothéose de Rameau: Orchestre philharmonique de Liège et ensemble Musiques Nouvelles, dirigés par Pierre Bartholomée", vol. 15, n 2 (2005), p. 101-104.

"Henri Pousseur: esquisses pour un visage", vol. 12, no 1 (2001), p. 9-16.

"Humeurs postmodernes", vol. 1, n ${ }^{\circ} 1$ (1990), p. 49-61.

"Jean Mozart Lesage ou une boussole pour dériver", vol. 18, n 2 (2008), p. 95-118.

"Jeu de raclures", vol. 8, nº 1 (1997), p. 9-17.

"L'éloge de la différence", vol. 7, n 1 (1996), p. 67-70.

"Le charme étrange des espaces familiers - Périple analytique dans Treppenmusik de John Rea ", vol. 11, $\mathrm{n}^{0} 1$ (2000), p. 9-32.

"Vous avez dit musique nouvelle?", vol. 9, nº 1 (1998), p. 53-57.

\section{GoNNEVILLE, Michel (animateur)} "Table ronde autour de Pousseur", vol. 12, n ${ }^{0} 1$ (2001), p. 29-42.

\section{Gonneville, Michel, HyLand, Marc,} et Ror, Stéphane

"Héritages", vol. 9, nº 1 (1998), p. $59-87$.

\section{GougEON, Denis}

"Garant communicateur, ou la parole agissante", vol. 7, nº 2 (1996), p. 27-35.

\section{GUÉRIN, François}

"Aperçu du genre électroacoustique au Québec, vol. 4, nº 1-2 (1993), p. 9-31.

\section{HARVEY, Jonathan}

"How do I compose?: Reflections on Wagner's Dream", vol. 18, nº 1 (2008), p. 38-43.

"Spatialization as a Compositional Tool and Individual Access to Music in the Future". Entretien réalisé par Nicolas Donin, vol. 16, nº 3 (2006), p. 75-81. 


\section{HenNion, Antoine}

"Les usagers de la musique: l'écoute des amateurs", vol. 14, nº 1 (2004),

p. 19-31.

\section{HÉTu, Jacques}

"Variations et variantes", vol. $15, \mathrm{n}^{\circ} 1$ (2004), p. 19-25.

\section{HÉTu, Joane}

"Itinéraire à compléter", vol. $6, \mathrm{n}^{\circ} 2$ (1995), p. 9-10.

\section{HYDE, Joseph}

"Off the Map? Sonic Art, the New Media Landscape”, vol. 13, n 3 (2003), p. $33-40$.

\section{HYLAND, Marc}

"Amériques", vol. 4, n 1-2 (1993), p. 162-164.

"Improvisations", vol. 6, $\mathrm{n}^{\circ} 2$ (1995), p. 67-69.

"Propos et contredanses. Entrevue imaginaire", vol. 10, nº 1 (1999), p. 11-16.

\section{IMBERTY, Michel}

"Langage, musique et cognition Quelques remarques sur l'évolution nécessaire des problématiques psychologiques des vingt dernières années", vol. 13, nº 2 (2003), p. 93-109.

\section{JAUJOU, Nicolas}

"Commerces discographiques Rayons de disques, conversations et communauté", vol. 14, no 1 (2004), p. 33-41.

\section{JONES, Pamela}

"À la recherche des racines. La vie et la carrière d'alcides lanza en Argentine", vol. 10, n 2 (1999), p. 9-16.

\section{KALTENECKER, Martin}

"La satire et l'épître", vol. 16, nº 1 (2006), p. 9-31.
KendeRGI, Maryvonne

"Serge Garant, toujours présent ", vol. $7, n^{\circ} 2$ (1996), p. 12-13.

\section{KLÜPPELHOLZ, Werner}

"Opéras sans chant: les films de Mauricio Kagel", vol. 3, nº 2 (1992), p. $45-63$.

\section{L'ÉCUYER, Sylvia}

"Le laboratoire musical de l'Université Simon Fraser - Une entrevue avec Barry Truax", vol. 19, n³ (2009), p. 49-57.

\section{LA RocheLLE, Réal}

"L'opéra audiovisuel dans le cinéma québécois - Vecteur de l'expérimentation sonore", vol. 19, $\mathrm{n}^{\circ} 3$ (2009), p. 67-76.

\section{LACASSE, Serge}

"La musique pop incestueuse - Une introduction à la transphonographie", vol. 18, nº 2 (2008), p. 11-26.

\section{LACHENMANN, Helmut}

"Composer dans l'ombre de Darmstadt (1987)", Yves Saint-Amant (trad.), vol. 15, nº 3 (2005), p. 53-66.

"Hearing [Hören] is Defenseless without Listening [Hören]", Derrick Calandrella (trad.), vol. 13, $\mathrm{n}^{\circ} 2$ (2003), p. 27-49.

"De la musique comme situation. Entretien avec Helmut Lachenmann". Entretien réalisé par Abigail Heathcote, Yves Saint-Amant (trad.), vol. 17, $\mathrm{n}^{\circ} 1$ (2007), p. 79-91.

\section{LAGACÉ, Claude}

"Musique tonale et musique d'aujourd'hui", vol. 7, nº 1 (1996), p. 36-39.

\section{LALONDE, Alain}

"De lui à moi à vous", vol. 3, n 1 (1992), p. 41-43.

\section{LANZA, alcides}

"alcides lanza's Musical Awakening in Buenos Aires. An Interview". Entretien avec Jonathan Goldman, vol. 17, $\mathrm{n}^{\circ} 2$ (2007), p. 83-91.

Lauber, Anne, Daveluy, Raymond, LAURIN, Rachel, et PAYETTE Alain "Réplique en sol majeur", vol. 7, n 1 (1996), p. 65-67.

\section{LAUXEROIS, Jean}

"À bon entendeur. Petite note sur "l'écoute structurelle" ", vol. 14, n 1 (2004), p. 87-102.

\section{LEBLANC, Jimmie}

"Denys Bouliane, maître de jeu", vol. 17, $n^{\circ} 3$ (2007), p. 93-117.

\section{LEDroIt, Christien}

"New Ground with Esprit Orchestra", vol. 13, $n^{\circ} 3$ (2003), p. 109-116.

\section{LEFEBVRE, Marie-Thérèse}

"Bibliographie chronologique des textes et travaux consacrés à Gilles Tremblay", vol. 6, n 1 (1995), p. 57-60.

"Chronologie des articles, entrevues et commentaires radiophoniques" [de Gilles Tremblay], vol. 5, nº 1 (1994), p. $73-76$.

"Complément bibliographique (19801996)" [à propos de Serge Garant], vol. 7, nº 2 (1996), p. 81-84

"Micheline Coulombe Saint-Marcoux et Marcelle Deschênes - Pionnières dans le sentier de la création électroacoustique", vol. 19, nº 1 (2009), p. $23-41$

"Repères biographiques et catalogue des œuvres", vol. 5, nº 1 (1994), p. $69-72$.

"Serge Garant - Documentation: pour débusquer l'inconnu. Chronologie de Serge Garant ", vol. 7, nº 2 (1996), p. 57-73. 
"Trois installations, deux environnements, une expérience", vol. 1, no 2 (1991), p. 27-28.

Éditorial [du numéro "Gilles Tremblay: réflexions"], vol. 5, nº 1 (1994), p. 5-7.

Éditorial [du numéro "Tremblay/ Varèse/Messiaen"], vol. 6, nº 1 (1995), p. 5-7.

LELONG, Guy

"Musique, in situ", vol. 17, $\mathrm{n}^{\circ} 3$ (2007), p. 11-20.

\section{LÉONARD, Robert}

"L'aventure et la provocation", vol. 8, nº 2 (1997), p. 21-23.

\section{LEROUX, Georges}

"Unité et diversité du savoir musical. À propos de Musiques. Une encyclopédie pour le XXI siècle, vol. 2 : "Les savoirs musicaux" ", vol. 16, no 1 (2006), p. 121-128.

\section{LEROUX, Robert}

"Musiques pour percussion", vol. 3, nº 1 (1992), p. 97-99.

"Serge Garant, chef d'orchestre, ou l'initiation exigeante", vol. $7, \mathrm{n}^{\circ} 2$ (1996), p. 23-26.

\section{LESAGE, Jean}

"Avant-dire. De l'utopie à la débrouille: parcours d'une génération", vol. 8, nº 1 (1997), p. 5-7.

"Claude Vivier, Siddhartha, Karlheinz Stockhausen, la nouvelle simplicité et le râga ", vol. 18, nº 3 (2008), p. 107-120.

"Non serviam", vol. 3, n 1 (1992),

p. 47-49.

"Réflexions en marge d'un festival", vol. 1, n 2 (1991), p. 39-42.

Lesage, Jean, et Perrin, Catherine "Entretien", vol. 15, no 1 (2004), p. 9-17.

\section{LeVesque, Patrick}

"L'élaboration du matériau musical
Claude Vivier", vol. 18, nº 3 (2008), p. 89-106.

\section{LIGETI, György}

"Sur la musique de Claude Vivier", vol. 2, no 1-2 (1991), p. 7-15.

\section{LIZÈRE, Emmanuelle}

"Tigouli : la création à inventer", vol. 16, n ${ }^{\circ} 2$ (2006), p. 57-67.

\section{LORTAT-JACOB, Bernard}

"L'oreille jazz : essai

d'ethnomusicologie", vol. 14, n 1

(2004), p. 43-51.

\section{LUSSIER, René}

"Collage", vol. 3, n 1 (1992), p. 44-46.

\section{Maheux, Diane}

"Deux décennies électroacoustiques.

Bilan comparatif ", vol. 4, no 1-2 (1993), p. 139-143.

\section{MARANDOLA, Fabrice}

"Pulau Dewata. Des arrangements raisonnables?", vol. 18, nº 3 (2008), p. 53-72.

\section{MARCo, Jean-Dominique}

"Le festival Musica et le Réseau Varèse", vol. 14, no 2 (2004), p. 59-66.

\section{MARCOTTE, Gilles}

"Deux ou trois malentendus", vol. 7 , $n^{\circ} 1$ (1996), p. 74-77.

\section{MARMARAS, Nicolas V.}

"L'interaction compositeur-ordinateur il y a 25 ans", vol. 18, n 1 (2008), p. 109-120.

\section{MASINO, Nicolas}

"Frank Zappa devant l'éternel: postérité problématique d'un iconoclaste impénitent ", vol. 14, n 3 (2004), p. 65-71.

"Requiem pour une fin de siècle", vol. 7, $n^{0} 1$ (1996), p. 33-36.

\section{MATHER, Bruce}

"Jouer et rejouer la musique de Garant", vol. 7, nº 2 (1996), p. 11-12.

"Musique à l'université Laval", vol. 3 , nº 1 (1992), p. 100-102.

\section{MCKInLEY, Maxime}

"Cage en liberté: réflexions libres", vol. 13, n 3 (2003), p. 103-107.

"Rencontre Haydn/Wagner dans les Berliner Momente de Walter Boudreau ", vol. 17, nº 1 (2007), p. 93-113

"Ultime édition de Rien à voir Entretien avec Francis Dhomont sur l'avenir acousmatique", vol. $16, \mathrm{n}^{\circ} 3$ (2006), p. 119-124.

\section{Menezes, Flo}

"La voie du syncrétisme - Sur la musique électroacoustique au Brésil", vol. $17, n^{\circ} 2$ (2007), p. 55-64.

\section{Mersy, Colette}

"Qu'est-ce que le postmodernisme musical?", vol. 1, nº 1 (1990), p. 9-25.

\section{Messier, Anne Marie}

"Ticket pour la liberté - Entretien avec Otto Joachim ", vol. 19, nº 3 (2009),

p. 39-47.

\section{MijnheER, Jaco, et DESJARDins, Thérèse}

"La chronologie des œuvres de Claude Vivier. Historisation de la déshistoire", vol. 2, n 1-2 (1991), p. 17-29.

\section{MoLINo, Jean}

"Classiques et classicisme à l'âge postmoderne", vol. 1, nº 1 (1990), p. $75-81$.

\section{MORAND, Louise}

"La morale de l'histoire...Quelques horizons esthétiques de Frank Zappa", vol. 14, $\mathrm{n}^{\circ} 3$ (2004), p.73-89. 
MOREL, François

"En Mémoire: esquisse d'une rencontre", vol. 7, n 2 (1996), p. 9-10.

\section{MORIN, Éric}

"Foi et persévérance. Les 75 ans de François Morel", vol. 12, nº 1 (2001), p. 96-99.

\section{Mumma, Gordon}

"An Electronic Music Studio For the Independent Composer [1964]", vol. 19, $n^{0} 3$ (2009), p. 85-90.

\section{NATTIEZ, Jean-Jacques}

"Apories du relativisme.

Considérations intempestives... et inactuelles", vol. 6, n 2 (1995), p. 61-65.

"De quelle tonalité parlez-vous?", vol. 9, $\mathrm{n}^{0} 1$ (1998), p. 47-51.

"Éphémérides d'une tournée", vol. 3 , n 1 (1992), p. 55-76.

"Faut-il tout accepter?", vol. 1, n 2 (1991), p. 43-49.

"Le quatuor à cordes: un genre dépassé?", vol. 11, nº 2 (2000), p. $73-76$

"Pauline Vaillancourt: la voix nue", vol. 3, nº 1 (1992), p. 107-108.

"Quelques problèmes de la musicologie critique selon Deliège", vol. 16, nº 1 (2006), p. 73-88.

Éditorial [du numéro "Boulez au Canada. Portrait d'impact"], vol. 3, $\mathrm{n}^{\circ} 1$ (1992), p. 5-6.

Éditorial [du numéro "Carte blanche à Bouliane et Rea"], vol. 9, nº 2 (1998), p. 5-6.

Éditorial [du numéro "Claude Vivier"], vol. 2, nº 1-2 (1991), p. 5-6.

Éditorial [du numéro "L'air du temps"], vol. 9, n 1 (1998), p. 5-7.

Éditorial [du numéro " Montréal musiques actuelles"], vol. 1, $\mathrm{n}^{\circ} 2$ (1991), p. 5-6.
Éditorial [du numéro "Musique actuelle?»], vol. 6, nº 2 (1995), p. 5-8.

Éditorial [du numéro "Opéra? Gauvreau, Provost, Kagel "], vol. 3, n ${ }^{\circ} 2$ (1992), p. 5-6.

Éditorial [du numéro

"Postmodernisme"]", vol. 1, n ${ }^{0} 1$ (1990), p. 5-7.

Éditorial [du numéro "Ruptures?"], vol. 7, nº 1 (1996), p. 5-7.

Introduction [à la réédition des cours d'été de Darmstadt], vol. 15, n 3 (2005), p. 23.

\section{Nicholson, Georges}

"Cage par lui-même - Entrevue avec John Cage", vol. 8, nº 2 (1997), p. 7-17.

\section{Nicolas, François}

"L'exigence du vouloir chez Deliège. À propos du livre de C. Deliège, Cinquante ans de modernité musicale: de Darmstadt à l'Ircam ", vol. 16, n 1 (2006), p. 33-44.

\section{NORMANDEAU, Robert}

"... et vers un cinéma pour l'oreille", vol. 4, no 1-2 (1993), p. 113-125.

"Faux et usage de faux. Réponse aux mélodistes indépendants", vol. 8, n 1 (1997), p. 77-79.

"Influence de Stockhausen chez les compositeurs électroacoustiques québécois", vol. 19, nº 2 (2009), p. 51-56.

"Qu'est-ce concert?", vol. 13, nº 1 (2002), p. 43-50.

\section{Nouss, Alexis}

"En dire plus", vol. 12, nº 2 (2002), p. 69-70.

NovoA, Laura (éd.)

"Document. Alberto Ginastera au Centro Latinoamericano de Altos Estudios Musicales (CLAEM). Correspondance 1961-1970", vol. 17, $n^{0} 2$ (2007), p. 35-41.

\section{OLDS, David}

"De Toronto : la diversité des styles", vol. 4, no 1-2 (1993), p. 33-37.

"Un atelier de Boulez: visite guidée de Mémoriale", vol. 3, nº 1 (1992), p. 83-92.

\section{OLIVIER, Dominique}

"Montréal (1991-1992). Vigueur accrue ou derniers soubresauts d'une civilisation", vol. 3, nº 2 (1992), p. 81-97.

"Montréal (1994-1995). L'accessibilité comme nouveau critère esthétique", vol. 7, nº 1 (1996), p. 93-109.

"Montréal. Portrait d'une saison hybride (1990-1991)", vol. 2, no 1-2 (1991), p. 163-177.

"Musique actuelle et ambiguiité", vol. 6, nº 2 (1995), p. 35-38.

"Positions. La mécanique des ruptures", vol. 7, nº 1 (1996), p. 17-18.

"Saison 1992-1993. L'année de la nostalgie", vol. 5, nº 1 (1994), p. 79-81.

"Saison 1993-1994. Le développement de l'individualisme, première conséquence du désengagement", vol. 6, nº 1 (1995), p. 65-80.

\section{OSWALD, John}

"Le plunderphonique, ou le piratage audio comme prérogative compositionnelle", Guy Marchand (trad.), vol. 18, nº 2 (2008), p. 27-38.

\section{OUZOUNIAN, Gascia}

"Visualizing Acoustic Space", vol. 17, $\mathrm{n}^{0} 3$ (2007), p. 45-56.

\section{Palardy Roger, Danielle}

"Dans le ventre de la musique actuelle", vol. 6, nº 2 (1995), p. 11-13.

\section{PALlandRe, Jean}

"La charpente invisible (une pratique expérimentale de la phonographie)", vol. 13, nº 3 (2003), p. 67-73. 
PAnNeton, Isabelle

"Kopernikus. Entrevue avec Thom

Sokoloski ", vol. 12, n 2 (2002),

p. 45-50.

"À la manière des préludes non mesurés", vol. 8, nº 1 (1997), p. 27-30.

"L'après-rupture", vol. 7, no 1 (1996), p. 29-33.

"La musique de Serge Garant", vol. 4, no 1-2 (1993), p. 157-162.

PASCAL, Noémie,

"Identités dans un monde de pluralité. Musique(s) et société(s) au tournant du xxI ${ }^{\mathrm{e}}$ siècle" ", vol. $15, \mathrm{n}^{\circ} 2$ (2005), p. 55-72.

"L'imagination au pouvoir. Portrait de la création musicale pour jeune public (Québec, 1990-2005)", vol. 16, nº 2 (2006), p. 21-41.

"Sur la trace des companion pieces.

Ébauche", vol. 18, nº 2 (2008), p. 61-75.

\section{PAuset, Brice}

"Das Einfachste, schwer zu machen [Bertolt Brecht]", vol. 15, n 3 (2005), p. 71-72.

\section{Pelinski, Ramon}

"Masque de l'identité. Réflexions sur Exotica de Mauricio Kagel ", vol. 6, $\mathrm{n}^{\circ} 2$ (1995), p. 47-59.

\section{Pelletier, Marie}

"Sans titre", vol. 7, nº 1 (1996), p. 73-74.

\section{Pennycook, Bruce}

"La musique électroacoustique en concert. Vieux problèmes, nouvelles solutions", vol. 4, n 1-2 (1993), p. 93-102.

\section{PÉPIN, Clermont}

"Jean Papineau-Couture, hommage d'un collègue", vol. 12, nº 1 (2001), p. 100.

\section{Perron, Alain}

"Panorama", vol. 10, nº 1 (1999), p. 41-51.

\section{PICARD, Isabelle}

"Ana Sokolovic", vol. 16, ñ 2 (2006), p. 105-110.

"Biographie d'André Villeneuve", vol. 15, n² 2 (2005), p. 93-95.

"Liste des œuvres d'André Villeneuve", vol. 15, n' 2 (2005), p. 97-99.

"CEuvres principales d'Ana Sokolovic", vol. 16, $n^{\circ} 2$ (2006), p. 111-113.

\section{PICHÉ, Jean,}

"De la musique et des images", vol. 13, $n^{\circ} 3$ (2003), p. 41-49.

"De Montréal: l'explosion d'une communauté", vol. 4, nº 1-2 (1993), p. 51-54.

"Musiques en Cage", vol. 8, nº 2 (1997), p. 25-31.

"New Music America. Contexte et rationalisation", vol. 1, n 2 (1991), p. 7-8.

"Non! Mais acceptons que le jardin soit plus grand que vous ne le croyez!", vol. 1, nº 2 (1991), p. 51-54.

"Nouvelle musique improvisée", vol. 3, no 1 (1992), p. 102-103.

"Vers une mécanique de l'imaginaire... Réflexions étoilées sur la composition algorithmique", vol. 4, no 1-2 (1993), p. 103-111.

\section{Portugais, Jean}

"Le Quatuor selon Schafer... et selon d'autres", vol. 11, n 2 (2000), p. 7-9.

"Présentation" [de la table ronde sur les quatuors à corde], vol. 11, $\mathrm{n}^{\circ} 2$ (2000), p. 55-57.

\section{Portugais, Jean, et RANZENHOFER, Olga}

"Îles de la nuit. Parcours dans l'œuvre pour quatuor à cordes de R. Murray Schafer", vol. 11, n² 2 (2000), p. 15-52.

\section{Pousseur, Henri}

"“Créer" : réponse ou défi aux

savoirs?", vol. 12, n 1 (2001), p. 73-85.

"Autobiographie", vol. 12, nº 1 (2001), p. 7.

"Visages de Pousseur". Entrevue réalisée par Michel Gonneville, vol. 12, $\mathrm{n}^{0} 1$ (2001), p. 19-27.

\section{Prévost, Hélène}

"De la musique de geste à la musique du son. De SuperMémé à I8U ", vol. 19, no 1 (2009), p. 71-81.

"Musique actuelle, qu'est-ce à dire?", vol. 6, nº 2 (1995), p. 31-33.

\section{Prieto, Eric}

"Speech Melody and the Evolution of the Minimalist Aesthetic: Steve Reich's The Cave", vol. 12, nº 2 (2002), p. 21-42.

\section{Provost, Serge}

"Improvisations, pop, rock et autres. Les concerts du Spectrum et d'ailleurs", vol. 1, n 2 (1991), p. 28-31. "L'écriture... plus actuelle que jamais!", vol. 6, no 2 (1995), p. 39-46.

"L'œuvre de Serge Garant, ou la musique de la sensibilité secrète", vol. 7, no 2 (1996), p. 15-21.

"Le mot, le son, le sens", vol. $3, \mathrm{n}^{\circ} 2$ (1992), p. 21-41.

"Le quatuor à cordes comme lieu de désir", vol. 11, nº 2 (2000), p. 59-61.

"Lire Xenakis. À propos du recueil d'écrits de Xenakis. Kéleüta", vol. 5, $n^{\circ} 2$ (1994), p. 69-75.

"Transmigration, du son vers la forme", vol. 8, n 1 (1997), p. 43-48.

Éditorial [du numéro "Espace Xenakis"], vol. 5, no 2 (1994), p. 5-7.

\section{RACINE, Rober}

"Cage/Satie: le silence vertigineux. Quelques rencontres avec John Cage sans jamais l'avoir regardé dans les yeux", vol. 8, nº 2 (1997), p. 39-43. 


\section{RADFORD, Laurie}

"Hommage à Bengt Hambreus", vol. 10, nº 2 (1999), p. 51-54.

"Le meilleur professeur est un éternel étudiant. Bengt Hambreus, compositeur et pédagogue", vol. 10, no 2 (1999), p. 29-38.

\section{Ramaut-Chevassus, Béatrice} " "L'évolution historique" et ses marges. Éléments pour une lecture critique de Cinquante ans de modernité musicale de Deliège", vol. 16, n ${ }^{0} 1$ (2006), p. 65-72.

\section{RATTÉ, Michel}

"Erratum. Le statut socio-institutionnel de la musique savante", vol. 8, no 1 (1997), p. 71-76.

"Le statut socio-institutionnel de la musique savante", vol. 7, nº 1 (1996), p. $25-29$.

\section{REA, John}

"Darmstadt as Refectory (Mess Hall)", vol. 15, $n^{0} 3$ (2005), p. 73-77.

"Gradus ad Infernum: Entrevue avec Ferdinand Larven Niemantz, "penseur de la musique" et auteur de Musical Compositions of the Century", vol. 9 , $\mathrm{n}^{0} 2$ (1998), p. 9-23.

"In nomine Zappa, Zappatore: Sketch of an Hypothesis (S/Z2) + (sv/gv)", vol. 14, $\mathrm{n}^{0} 3$ (2004), p. 103-111.

"Ite, Symphonia est. Apostille au nom de la collectivité musico-religieuse", vol. 11, $\mathrm{n}^{0} 3$ (2001), p. 21-31.

"La Liberté guidant le peuple", vol. 3 , $\mathrm{n}^{\circ} 1$ (1992), p. 49-50.

"Le quatuor à cordes est une forme tabou, un fossile et il n'appartient plus à une tradition vivante", vol. $11, \mathrm{n}^{\circ} 2$ (2000), p. 63-66.

"Les œuvres de Mario Bertoncini", vol. 15, n 1 (2004), p. 105-107.
"Nashville ou Darmstadt. Le masque mortuaire de la postmodernité", vol. $9, n^{\circ} 2$ (1998), p. 61-73.

“On Stockhausen's Kontakte (1959-60) for tape, piano and percussion. A Lecture/Analysis (1968)", vol. 19, nº 2 (2009), p. 77-86.

"Portrait de Mario Bertoncini. Compositions et autres choses "inutiles" à propos de la carrière de Mario Bertoncini ", vol. 15, nº 1 (2004), p. 87-92.

"Portrait du compositeur en jeune poète", vol. 9, nº 1 (1998), p. 41-46.

"Postmodernité "que me veux-tu" ", vol. 8, nº 1 (1997), p. 55-69.

«Reflets dans l'eau... bénite. Douze images impures: la vie et la musique de Claude Vivier", vol. 1, n 2 (1991), p. 71-79.

"The Sorcerer as Apprentice: his Notes. The computer in/and teaching the craft of composition", vol. 18, n ${ }^{0} 1$ (2008), p. 73-91.

\section{ReNAUd, Lucie}

"La création pour jeune public: camaïeu de styles musicaux", vol. 16, $n^{\circ} 2$ (2006), p. 43-56.

\section{REVERDY, Michèle}

" "Qu'est-ce que vous faites dans la vie?" "Je suis compositrice..." ", vol. 19, n० 1 (2009), p. 15-21.

\section{RHÉAUME, Martine}

"Évolution d'un style musical. Comment Vivier passe-t-il d'une œuvre à l'autre?", vol. 18, n 3 (2008), p. 73-88.

"Réflexions sur les festivals, la musique contemporaine et l'identité culturelle québécoise", vol. 15, n 2 (2005), p. 73-82.

\section{RICARD, Gisèle}

"Dans le sillage de Nil Parent:

Smeul et Gimel. Points de repère dans l'histoire de la musique électroacoustique à Québec", vol. 19, $n^{0} 3$ (2009), p. 59-65.

\section{RIVEST, Johanne}

"Atlas Eclipticalis, commande montréalaise", vol. 8, nº 2 (1997), p. 61-69.

"Claude Vivier: les œuvres d'une discographie imposante", vol. 2, nº 1-2 (1991), p. 137-161.

"Improviser ou interpréter Theatre Piece? Entrevue avec Malcolm Goldstein ", vol. 8, no 2 (1997), p. 53-60.

"Présence audible : La musique enregistrée de Serge Garant ", vol. 7, $n^{\circ} 2$ (1996), p. 80.

Éditorial [du numéro "Québécage»], vol. 8, nº 2 (1997), p. 5-6.

\section{ROBERT, Véronique}

«Prologue pour les écrits d'un compositeur " [à propos de Claude Vivier], vol. 2, n 1-2 (1991), p. 31-37.

\section{RoGALSKY, Matt}

"Finger Exercises for Oscillators: István Anhalt on Electronic Music", vol. 19, $\mathrm{n}^{0} 3$ (2009), p. 77-84.

\section{Rogers, Stephen}

"Travelogue pour un Marco Polo [My Travels with Claude?]. A journey through the composer's life and work in 10 days", vol. 18, n 3 (2008), p. $27-51$

\section{Roy, Stéphane}

"Analyse des œuvres acousmatiques : Quelques fondements et proposition d'une méthode", vol. 4, nº 1-2 (1993), p. 67-92.

"Francis Dhomont: deux disques pour un anniversaire", vol. 3, n 1 (1992), p. 119-125.

"L'Utopie, contrée fertile de l'aventure électroacoustique", vol. 13, n 3 (2003), p. 19-31. 
"Les concerts de musique

électroacoustique à l'Acousmonium ", vol. 1, nº 2 (1991), p. 19-22.

\section{RugelEs, Alfredo}

"Le rapport entre la musique contemporaine vénézuélienne dite "savante" et les musiques populaire et folklorique", vol. 17, n 2 (2007), p. $43-53$.

\section{SALLIS, Friedemann}

"Fleurs recyclées. Sur les traces de relations souterraines dans l'Officium breve, memoriam Andrea Szervánszky opus 28 pour quatuor à cordes de György Kurtág", vol. 18, nº 1 (2008), p. $45-58$.

"Le paradoxe postmoderne et l'œuvre tardive de Luigi Nono", vol. 11, $\mathrm{n}^{0} 1$ (2000), p. 69-84.

\section{SAVOURET, Alain}

"Électroacoustique et perspective phonoculturelle", vol. 13, $\mathrm{n}^{\circ} 1$ (2002), p. 9-19.

\section{SCHAFER, R. Murray}

"Acoustic Space", vol. 17, nº 3 (2007), p. $83-86$.

"La "quaternité" et le quatuor", vol. 11, nº 2 (2000), p. 11-13.

\section{SiCiliano, Giancarlo et Drouin, Jacques \\ "Les concerts présentés aux Foufounes électriques", vol. 1, n 2 (1991), p.22-24.}

\section{SMoje, Dujka}

"Les crises du modernisme dans l'histoire musicale", vol. 7, nº 1 (1996),

\section{Solomos, Makis}

"Les trois sonorités xenakiennes", vol. 5, nº 2 (1994), p. 21-39.

\section{Soucr, Chantal}

"Les spectacles de danse", vol. 1, $\mathrm{n}^{\circ} 2$ (1991), p. 25-27. p. 83-91.

\section{SOULEZ, Antonia}

"Phrases musicales. La musique dans la philosophie de Wittgenstein", vol. $17, n^{\circ} 1$ (2007), p. 27-47.

\section{SPRENGER-OHANA, Noémie}

"L'évolution d'un projet compositionnel en relation avec son environnement. Autour de Concerto d'Elvio Cipollone", vol. 18, n 1 (2008), p. 93-108.

\section{STÄBLER, Gerhard}

"Sans titre", Yves Saint-Amant (trad.), vol. $15, n^{\circ} 3$ (2005), p. 79-81.

\section{Stadlen, Peter (introduction de Nicolas Donin)}

"Le malentendu pointilliste", Yves Saint-Amant (trad.), vol. 15, nº 1 (2004), p. 27-39.

\section{Steenhuisen, Paul}

"Darmstadt 1992: The Understory", vol. $15, n^{\circ} 3$ (2005), p. 83-86.

"The Splendid Allure of Europium at the Inaugural Montréal/Nouvelles Musiques", vol. 14, no 2 (2004), p. 53-58.

\section{StÉvance, Sophie}

"La composition musicale et la marque du genre. L'examen conscient de l'"écriture féminine" ", vol. 19, n ${ }^{\circ} 1$ (2009), p. 43-55.

"La Dream House ou l'idée de la musique universelle", vol. $17, \mathrm{n}^{\circ} 3$ (2007), p. 87-92.

\section{Stockhausen, Karlheinz}

"La mesure du temps. Un entretien inédit avec Stockhausen (1958)". Entretien réalisé par Maryvonne Kendergi, Réjean Beaucage (transcr.), vol. 19, $n^{\circ} 2$ (2009), p. 63-75.

\section{SwIFT, Daniel}

"Actuel/temporel/virtuel", vol. 6, n 2 (1995), p. 19-21.

\section{SYKES, Claire}

"Henry Brant et sa musique", Guy Marchand (trad.), vol. 17, $n^{\circ} 3$ (2007), p. 77-82.

SzEndy, Peter et Donin, Nicolas "Otographes", vol. 13, n 2 (2003), p. 11-25.

\section{TAGG, Philip}

"Musiques. Une encyclopédie pour le XxI siècle, vol. 1: "Musiques du Xx" siècle". Compte rendu ", vol. 16, no 1 (2006), p. 97-119.

\section{TALPASH, Andriy}

"La parodie dans Osten de Mauricio Kagel ", Marc Hyland (trad.), vol. 12, $n^{\circ} 3$ (2002), p. 57-62.

\section{TeXIER, Marc}

"Les deux premiers disques du Nouvel Ensemble Moderne", vol. 3, nº 1 (1992), p. 104-107.

\section{ThÉBERge, Paul}

"Exploring the creative process: Hypermedia tools for understanding contemporary composition", vol. 18, $\mathrm{n}^{0} 2$ (2008), p. 77-85.

\section{StIEGLER, Bernard}

"L'armement des oreilles. Devenir et avenir industriels des technologies de l'écoute", vol. 16, nº 3 (2006), p. 33-42.

"Le circuit du désir musical.

L'interprète, le compositeur, l'auditeur - organes et instruments". Entretien réalisé par Nicolas Donin, vol. 15, $\mathrm{n}^{\circ} 1$ (2004), p. 41-56.

\section{ThIBAULt, Alain}

"Culture numérique et électroacoustique", vol. 13, n 1 (2002), p. 51-54.

\section{TIFFon, Vincent}

"Analyse électroacoustique", vol. 17, $n^{\circ} 3$ (2007), p. 119-123. 


\section{TORSTENSSON, Klas}

"Klas Torstensson in Conversation".

Entretien réalisé par Jonathan

Goldman, vol. 14, n 2 (2004), p. 49-52.

\section{Tousignant, François}

"Pour corriger quelques "erreurs" ", vol. 7, nº 1 (1996), p. 6o-63.

"Rendre à César..." vol. 3, n 1 (1992), p. 50-53.

\section{TREMBLAY, Gilles}

"Allocution à l'occasion de la remise des Prix du Québec 1991", vol. 5, nº 1 (1994), p. 61

"Acoustique et forme chez Varèse", vol. 6, nº 1 (1995), p. 23-35.

"Claude Vivier, en mémoire, en présence", vol. 5, nº 1 (1994), p. 63-65.

"Composer la musique contemporaine", vol. 5, $\mathrm{n}^{\circ} 1$ (1994), p. 10-17.

"Découvrir ce chant infiniment moderne", vol. 5, no 1 (1994), p. 37-46. "Émergence de la forme dans la matière. De Varèse à Mozart", vol. 6, no 1 (1995), p. 37-41.

"En toute amitié", vol. 7, nº 2 (1996), p. 10-11.

"Java et Bali", vol. 5, n 1 (1994), p. $25-27$.

"L’acte musical", vol. 5, no 1 (1994), p. 9.

"La fragilité du chant est sa force même", vol. 5, nº 1 (1994), p. 22-25.

"La musique après la fin de la musique", vol. 5, nº 1 (1994), p. 51-55.

"Le bruit. Prospective négative et prospective positive", vol. $5, \mathrm{n}^{\circ} 1$ (1994), p. 29-35.

"Le compositeur Gilles Tremblay a refusé l'Ordre du Canada", vol. 5, n ${ }^{0} 1$ (1994), p. 59-60.

"Les sons en mouvement (sur Varèse)", vol. 6, nº 1 (1995), p. 15-21.
"Les Vêpres de la Vierge", vol. 6, n¹ (1995), p. 51-55.

"Même la mort se vit", vol. 5, n 1 (1994), p. 21-22.

"Notes pour Cantique de durées", vol. 6, nº 1 (1995), p. 43-50.

"Serge Garant, être de rigueur et d'émotion", vol. 5, nº 1 (1994), p. 65-67.

"Silence et liturgie", vol. 5, n 1 (1994), p. 46-50.

"Silences, silence", vol. 6, n 1 (1995), p. 9-14.

"Une reconnaissance", vol. 5, $\mathrm{n}^{\circ} 1$ (1994), p. 19-21.

"Vers un choix (projets du pays qui vient)", vol. 5, n 1 (1994), p. 57-59.

\section{TREMBLAY, Jacques}

"L'écriture à haute voix: Lonely Child de Claude Vivier", vol. 11, n 1 (2000), p. $45-67$.

\section{TroTtIER, Danick}

"Compte rendu: Elliott Carter, A Centennial Portrait, Letters and

Documents", vol. 19, nº 3 (2009), p. 113118.

"Entrevue avec André Villeneuve sur l'enjeu de l'identité dans la poïétique musicale", vol. 15, nº 2 (2005), p. 83-91.

Éditorial [du numéro "Carte d'identités"] vol. 15, n 2 (2005), p. 7-12.

"La poïétique d’Yves Daoust. Entre représentation socioculturelle et questionnement identitaire", vol. 15, no 2 (2005), p. 13-36.

\section{TRUAX, Barry}

"Electroacoustic Music and the Digital Future", vol. 13, no 1 (2002), p. 21-26.

\section{TURCOTTE, Roxanne}

"La création électroacoustique et les jeunes... Une démarche sur mesure" vol. 16, n² 2 (2006), p. 73-81.
VAILLANCOURT, Lorraine

"La musique aujourd'hui dans tous ses éclats", vol. 9, nº 2 (1998), p. 75-80.

"Points de Départ. Lettre (inédite) à Lise Bissonnette, directrice du Devoir ", vol. 7, nº 1 (1996), p. 9-11.

\section{VAILLANCOURT, Pauline}

"Chanter, jouer: l'aventure de Chants libres". Propos recueillis par JeanJacques Nattiez, vol. 3, nº 2 (1992), p. 65-68.

\section{VALVERDE, Gabriel}

"Utopie et idéal dans la composition musicale aujourd'hui", vol. 9, n 1 (1998), p. 7-15.

\section{VERDIER, Véronique}

"Compte rendu. Gérard Grisey, Écrits ou l'invention de la musique spectrale", vol. 19, nº 3 (2009), p. 109-112.

\section{Vermaelen, Denis}

"Vue sur l'atelier d'Elliott Carter. À partir de Triple Duo", vol. 18, nº 1 (2008), p. 26- 31.

\section{VilleneuVe, André}

"Harmonies. Désert fertiles que j'interroge", vol. 10, nº 1 (1999), p. $63-72$.

\section{VINAY, Gianfranco}

"Vue sur l'atelier de Salvatore Sciarrino. À partir de Quaderno di Strada et Da Gelo a Gelo", vol. 18, n 1 (2008), p. 15-20.

\section{VINET, Hugues}

"Le projet SemanticHIFI. Manipulation par le contenu d'enregistrements musicaux", vol. 16, n 3 (2006), p. 65-73.

VIVIER, Claude,

"Liebesgedichte", vol. 2, n 1-2 (1991), p. 65-66. 
"Cinq chansons pour percussion ", vol. 2, no 1-2 (1991), p. 109-111.

"Glaubst du an die Unsterblichkeit der Seele", vol. 2, n 1-2 (1991), p. 133-135.

"Hiérophanie", vol. 2, n 1-2 (1991), p. 49.

"Journal", vol. 2, no 1-2 (1991), p. 79-87. "Kopernikus, opéra — rituel de mort", vol. 2, no 1-2 (1991), p. 95-107.

"Lettura di Dante", vol. 2, nº 1-2 (1991), p. 60-61.

"Lonely Child", vol. 2, n 1-2 (1991), p. 107-109.

"Love Songs", vol. 2, no 1-2 (1991), p. $87-88$.

"Mantra de Stockhausen", vol. 2, no 1-2 (1991), p. 93-95.

"Musik für das Ende", vol. 2, no 1-2 (1991), p. 53-55.

"Nanti Malam", vol. 2, n n 1-2 (1991), p. 88-89.

"O! Kosmos", vol. 2, n 1-2 (1991), p. 58-60.

"Ojikawa", vol. 2, no 1-2 (1991), p. 48.

"Orion", vol. 2, no 1-2 (1991), p. 121-123.

"Prologue pour un Marco Polo", vol. 2, no 1-2 (1991), p. 111-112.

"Pulau Dewata", vol. 2, n 1-2 (1991), p. 89.

"Shiraz", vol. 2, nº 1-2 (1991), p. 89.

"Trois airs pour un opéra imaginaire", vol. 2, no 1-2 (1991), p. 131-133.

"Wo Bist du Licht!", vol. 2, n 1-2

(1991), p. 119-120.
"Zipangu", vol. 2, ñ 1-2 (1991), p. 109.

"À propos de Gilles Tremblay", vol. 2, nº 1-2 (1991), p. 130-131.

"Cinéma et musique", vol. 2, nº 1-2 (1991), p. 127-129.

"Créativité et cinéma québécois", vol. 2, n 1-2 (1991), p. 118.

"De la critique musicale", vol. 2, n 1-2 (1991), p. 66-68.

"En musicant [sic]", vol. 2, no 1-2 (1991), p. 40-41.

"Est bien vu ici qui veut être médiocre", vol. 2, n 1-2 (1991), p. $64-65$.

"Imagine", vol. 2, no 1-2 (1991), p. 123125.

"Introspection d'un compositeur", vol. 2, nº 1-2 (1991), p. 92-93.

"Japon", vol. 2, no 1-2 (1991), p. 68-69.

"L'acte musical", vol. 2, n 1-2 (1991), p. 49-51.

"L'Amour", vol. 2, n 1-2 (1991), p. 41-42.

"La musique de la nouvelle génération", vol. 2, nº 1-2 (1991), p. 90-91.

"Le clown", vol. 2, n 1-2 (1991), p. 46-48.

"Musique", vol. 2, nº 1-2 (1991), p. 39-40.

"Noël", vol. 2, nº 1-2 (1991), p. 43-45.

"Not' petit bonheur", vol. 2, n" 1-2 (1991), p. 45-46.

"Notes du soir", vol. 2, n 1-2 (1991), p. 51-53.
"Petit historique de la musique contemporaine", vol. 2, n 1-2 (1991), p. 120-121.

"Postulat", vol. 2, no 1-2 (1991), p. 45. "Pour Gödel ", vol. 2, n 1-2 (1991), p. $125-126$.

"Que propose la musique?", vol. 2, no 1-2 (1991), p. 129-130.

"Quelques considérations sur la composition musicale", vol. 2, no 1-2 (1991), p. 61-64.

"Serge Bélisle", vol. 2, nº 1-2 (1991),

p. $42-43$.

"Situation de la musique contemporaine au Canada ", vol. 2, no 1-2 (1991), p. 113.

"Situation de la musique", vol. 2, no 1-2 (1991), p. 113-116.

"Trois lettres de Bali", vol. 2, n 1-2 (1991), p. 70-79.

"Une allocution", vol. 2, no 1-2 (1991), p. 116-118.

\section{WATSON, Ben}

"Frank Zappa's Legacy: Just Another Hoover?", vol. 14, nº 3 (2004), p. 35-44.

\section{XENAKIS, Iannis}

"Musique, espace et spatialisation". Entretien réalisé par Maria Harley, vol. 5, $n^{\circ} 2$ (1994), p. 9-20.

\section{Young, Gayle}

"Hugh Le Caine. De la saqueboute au multi-track", vol. 19, nº 3 (2009), p. 9-37. 